42 | 2012

La communication, dimension oubliée de l'intelligence économique

\title{
Le paradigme de classification et d'organisation des ressources humaines dans la radio-télévision publique espagnole : une proposition inachevée
}

Miguel Ángel Ortiz Sobrino

\section{OpenEdition}

Édition électronique

URL : http://journals.openedition.org/communicationorganisation/3980

DOI : 10.4000/communicationorganisation.3980

ISSN : $1775-3546$

Éditeur

Presses universitaires de Bordeaux

Édition imprimée

Date de publication : 1 décembre 2012

Pagination : $217-226$

ISBN : 978-2-86781-772-4

ISSN : 1168-5549

\section{Référence électronique}

Miguel Ángel Ortiz Sobrino, «Le paradigme de classification et d'organisation des ressources

humaines dans la radio-télévision publique espagnole : une proposition inachevée », Communication et organisation [En ligne], 42 | 2012, mis en ligne le 01 décembre 2014, consulté le 03 mai 2019. URL:

http://journals.openedition.org/communicationorganisation/3980 ; DOI : 10.4000/

communicationorganisation.3980 


\title{
Le paradigme de classification et d'organisation des ressources humaines dans la radio-télévision publique espagnole : une proposition inachevée.
}

\author{
Miguel Ángel Ortiz Sobrino'
}

Les professionnels et théoriciens de la communication sont unanimes à signaler que l'on traverse une étape de transformations radicales jamais vécues par la radio-télévision en Espagne. Dans les premières années de notre siècle, l'industrie audiovisuelle a subi un changement provoqué par trois motifs principaux : d'un côté, l'implantation de nouvelles formes de production et distribution des contenus favorisée par la convergence multimédia (Muro, 2010) ; de l'autre côté, la migration, en 2010, de la télévision analogique vers un modèle numérique qui a rendu possible un considérable accroissement du nombre de chaînes; et enfin, les changements subis par le système de financement de la radio-télévision publique espagnole, suite à la suppression de la publicité (des émissions) de télévision, en janvier 2010. Il est évident que cette transformation a eu des conséquences importantes dans la structure patronale, dans l'accroissement de l'offre et dans la redistribution du chiffre d'affaires du négoce radiophonique et de la télévision. Mais dans ce cas précis, il est important de ne pas perdre de vue comment ces trois facteurs ont modifié les profils professionnels et les plans de travail.

Selon A. Scolari, dans un article paru dans la revue Signo y pensamiento, l'application des TIC et la convergence des "mass media " ont affecté directement l'un des piliers fondamentaux qui soutient les entreprises : leurs Ressources Humaines (Scolari 2009). Du point de vue des entreprises, la convergence médiatique en Espagne a entraîné des bouleversements dans la réalisation des émissions d'information générale et de divertissement, grâce à l'utilisation de nouveaux outils technologiques et l'apparition de supports de diffusion numérique de pointe. Ces systèmes modernes de production audiovisuelle et les plates-formes émergentes relatives à la téléphonie mobile

1 Professeur titulaire, Université Complutense Madrid, Facultad de Ciencias de la Información, Président Association Radios Universitaires Espagnoles ; amortiz.@ccinf.ucm.es 
et à Internet, ont fait apparaître de nouveaux profils professionnels et des manières différentes d'organiser le travail dans le secteur (TV radio).

La structure de l'industrie audiovisuelle n'est pas étrangère à cette dimension technologique ni à l'impact sur ses travailleurs. La migration du système analogique de télévision vers un système numérique de TDT a contribué à changer son tissu industriel grâce à l'incorporation de nouvelles chaînes (Impulsa 2010-11). Selon l'Observatoire Audiovisuel Européen, un an plus tard, l'Espagne est le deuxième pays européen en nombre de chaînes de télévision (1180), seulement dépassé par la Grande Bretagne (1222). Face à cette nouvelle réalité, la recherche de synergies commerciales a impulsé la fusion entre les principales chaînes de télévision du pays, créant ainsi un système d'oligopole où les relations professionnelles ont changé. Cependant, ce bond entre la télévision analogique et la TDT a rendu possible l'apparition d'entreprises de télévision plus réduites, qui sont en majorité composées de jeunes travailleurs appartenant déjà à la dite "génération de natifs du numérique » familiarisés avec Web 2.0 et TIC.

Depuis longtemps, le système de financement, mis en cause, de la radio-télévision publique en Espagne conditionne sa dimension ainsi que ses méthodes de production et de relations de travail. Bien qu'en 2005 le Gouvernement espagnol ait approuvé la réduction d'un tiers des employés de la radio-télévision publique nationale afin d'assurer sa viabilité économique, actuellement les télévisions publiques régionales sont au centre du débat médiatique $^{2}$. Après les élections générales en Espagne, en novembre 2011, le Gouvernement, issu du triomphe du "Partido Popular ", redéfinit la structure de ces télévisions, dont la dette accumulée en 2011 s'est élevée aux environs de 2100 millions d'euros. Il s'agit d'un déficit insoutenable équivalant approximativement au budget du ministère de 1'Industrie. Selon les informations recueillies par les principaux moyens de communication, tout semble indiquer que cette nouvelle conception des autorités espagnoles de la radio-télévision publique régionale répond au dynamisme des modèles commerciaux et d'organisation "low cost», semblables à ceux installés dans le secteur privé de la radio et la télévision espagnole ${ }^{3}$ (López y Ortiz 2011). Il serait raisonnable de penser alors, que le futur du service public espagnol de radiodiffusion et télévision se dirige vers la création de centres de travail pourvus d'un nombre inférieur d'employés dont cependant on exigera de

2 En 2006 la radio-télévision espagnole a supprimé 4150 postes de travail. Une mesure qui faisait partie du plan de viabilité élaboré par le gouvernement du Président Rodriguez Zapatero. Aujourd'hui, le débat sur son soutènement reste actuel. Cf. El Mundo (14/01/2012) et El Economista (16/02/2012).

3 À partir de 2005, la réduction des structures d'organisation et des budgets d'exploitation est le pilier des modèles patronaux des nouvelles télévisions publiques régionales en Espagne, contrairement à ceux de la télévision publique de l'État et d'autres télévisions publiques régionales apparues pendant les deux dernières décennies du $\mathrm{XX}^{\mathrm{e}}$ siècle, et qui disposent de budgets considérables de production et d'une importante dotation technologique et de Ressources Humaines. 
plus grandes compétences professionnelles, afin d'assumer la diffusion des contenus multimédias.

\section{La mise en valeur de la polyvalence professionnelle dans la convergence médiatique}

Les dernières transformations subies par l'industrie de la radio-télévision en Espagne ont eu d'importantes conséquences sur le paradigme de ses profils professionnels. La portée de cet impact peut être observée aussi bien d'un point de vue de la convergence comme de la perspective d'un marché qui succombe, parfois, devant l'optimisation et la maximisation des bénéfices. Ce nouveau scénario de convergence médiatique a besoin, maintenant, de professionnels de la radio et la télévision qui dominent les TIC et les techniques de conception de contenus destinés à la diffusion grâce aux plates-formes multimédia. C'est-à-dire, des travailleurs "multi-supports » (polyvalents), capables d'accomplir leur travail avec l'esprit du web 2.0 et l'émergente web thématique 3.0. Actuellement, il n'y a aucun doute que sans la polyvalence fonctionnelle des travailleurs dotés de compétences professionnelles pour accomplir leurs tâches dans les différents circuits et environnements technologiques, les actuelles formes de commerce et de production seraient inimaginables (Rodríguez y Peinado, 2011). Alors, c'est pourquoi on pourrait exiger une valeur ajoutée due à la polyvalence des fonctions et la mobilité du travailleur qui rendent plus concurrentiels ces " média » et accordent aux professionnels une plus grande responsabilité dans la chaîne productive (Scolari, 2009 et Salaverría, 2003). Cette mise en valeur du concept " polyvalence » a favorisé une profonde mutation des profils professionnels audiovisuels et, surtout, le besoin de promouvoir la formation permanente pour les adapter à un environnement technologique vertigineusement changeant.

Une tout autre question enfin se pose dans certains cas si cette « polyvalence professionnelle » est interprétée par certaines entreprises du secteur selon les critères d'économie de coûts et précarité du travail, afin d'obtenir de plus grands bénéfices commerciaux ou d'abaisser les dépenses de production de contenus (Morillon ; Bouzon ; Cooren, 2009). En Espagne, cette tendance à l'optimisation de ressources et la philosophie "low cost » qui maintenant paraît dominer le secteur audiovisuel, s'est transformée en un facteur qui conditionne la production de la radio-télévision. Au point qu'au nom de cette soi-disant optimalisation de ressources commerciales, une fréquente détérioration des conditions de travail et de ressources s'est produite. Il semble alors évident que l'apparition, ces dernières années, de cette nouvelle mise en scène a favorisé que les entreprises de radio-télévision redéfinissent leur organisation fondée sur des profils professionnels polyvalents et sur la formation permanente de leurs travailleurs. 


\section{Le cas de RTVE, en Espagne}

Aucun des aspects mentionnés jusqu'alors n'aurait pu passer inaperçu par le groupe espagnol de radio-télévision appartenant à l'Etat, RTVE. La radio-télévision publique avait été pendant plusieurs années l'entreprise du secteur audiovisuel qui avait le plus souffert le manque de polyvalence des profils professionnels de ses employés. Pour cette raison, en octobre 2005, elle a mis en pratique une nouvelle réorganisation de ses Ressources Humaines dont les objectifs étaient, d'un côté, l'adaptation de ses travailleurs aux nouvelles exigences productives du nouveau scénario de convergence et, de l'autre, établir un procédé permettant de reconnaître et récompenser leur excellence professionnelle. Les méthodes obsolètes de production des contenus audiovisuels utilisés par l'entreprise jusque là et ses difficultés financières - la dette dépassait alors les 12000 millions d'euros - rendaient envisageable une telle mesure. La conséquence directe de cette initiative a entraîné une nouvelle définition de la gestion des Ressources Humaines, fondée sur un Plan de Carrières et un système de Promotion Professionnelle, où la gestion du talent et la formation permanente seraient le fondement de l'Organisation. Cette initiative s'est transformée en expérience pionnière dans le secteur audiovisuel espagnol.

Cependant, l'inertie historique ne rendait pas faciles les faits. Les relations collectives de RTVE reposaient sur la philosophie d'un règlement de relations du travail surgies dans les premières années de ce " Holding " espagnol ${ }^{4}$. La réglementation, jusqu'en 2005, des relations contractuelles des employés de RTVE établissait une structure de catégories professionnelles excessivement rigides et atomisées qui empêchait la polyvalence de ses travailleurs. Ainsi, la description des fonctions et compétences relatives à cette taxonomie du travail entravait l'adéquation de ses travailleurs au nouvel environnement technologique et aux nécessités du marché audiovisuel.

Ce cadre normatif n'apportait pas non plus les conditions adéquates pour une motivation correcte de ses groupes de travail. La concentration excessive des tâches que doit réaliser chaque travailleur oblige de manière systématique la RTVE à faire recours à des compléments salariaux de polyvalence et de mobilité fonctionnelle afin d'obtenir l'assouplissement et l'implication de ses professionnels dans l'Organisation. Le manque de critères objectifs pour l'attribution de ces compléments salariaux provoque certes un degré d'arbitraire car ceux-ci dépendaient, exclusivement, de la volonté de certains cadres de direction de l'entreprise. Ces circonstances créent un scénario où, évidemment, ont lieu des problèmes de concurrence, d'efficacité et, parfois, de confiance.

4 Le groupe RTVE est né en 1980, après la publication de la Loi sur le Statut de la Radio et Télévision en Espagne. À partir de cette Loi, les services de radio et de télévision propriété de l'État qui jusqu'alors avaient été des organisations indépendantes se sont intégrées dans un " holding " d'entreprises qui, sous la marque RTVE, a réuni « Radiocadena Española », « Radio Nacional de España » et «TVE ». 
La situation, s'il y a lieu, s'aggrave d'autant plus à cause du système d'élection des organes de Direction. Lincorporation fréquente des cadres externes de l'entreprise et la dépendance du pouvoir politique des premiers niveaux de décision de la RTVE publique, rendaient impossible une continuité de leurs stratégies corporatives. Il faut rappeler que, jusqu'en 2006, le Président Directeur Général de la Société était nommé directement par le gouvernement, parmi ses collaborateurs de confiance. Le consensus entre les partis politiques espagnols pour approuver une loi pour réglementer, à partir de ce moment, la nomination par majorité parlementaire du Président et du Conseil d'Administration, semblait balayer les difficultés vers de nouvelles stratégies à long terme. Ce consensus ne s'était jamais réalisé et ne se réalise toujours pas dans les accords-cadre en vigueur dans les autres entreprises de radio-télévision en Espagne nées à partir des années 80, dans le cas des télévisions publiques des autonomies ${ }^{5}$, et - à partir de $1989^{6}$ - dans les télévisions privées. Plusieurs de ces Compagnies de radio-télévision ont donné naissance à un régime de relations de travail qui leur a permis une concurrence et une opérativité améliorées. C'est pourquoi la situation a exigé une nouvelle manière de gérer les Ressources Humaines de la radio-télévision publique de l'État et de les adapter à une scène audiovisuelle changeante, beaucoup plus concurrentielle et technologique (Ortiz Sobrino, 2011).

\section{Un modèle de classification et d'organisation de la radio-télévision basé sur la gestion du talent et la formation continue}

La reformulation de la gestion de ses Ressources Humaines se produit vers la fin de 2005, lorsque RTVE propose un Plan de Promotion Professionnel et un Plan de Carrières Professionnelles, dont le premier était destiné à tous les employés de l'Organisation alors que le second touchait seulement les professionnels considérés d'un potentiel plus élevé de direction. Il s'agissait non seulement d'un outil pour optimiser l'organisation de ses processus productifs, mais aussi d'un instrument pour le développement professionnel des travailleurs de la radio-télévision publique. Doter l'entreprise d'un nouveau système de classification professionnelle fondé sur des catégories et profils professionnels plus polyvalents, où le travailleur pourrait avoir de l'avancement professionnel et financier, a été l'objectif principal de cette proposition. Dans ce Plan de Promotion Professionnel proposé par RTVE, un salaire de base et différents compléments salariaux pourraient être attribué

5 Les stations publiques régionales de radio-télévision ont commencé à émettre, à partir de 1983, grâce à l'entrée en vigueur de la Loi du Troisième Canal. Ces stations appartiennent, actuellement, aux différentes Communautés Autonomes d'Espagne, face à RTVE qui appartient à l'État Central. Dorénavant, ces émissions de radio-télévisions publiques ont commencé à cohabiter avec la radio-télévision publique : TVE et Radio Nacional de España. Les premières émissions de TVE ont commencé en 1956 et celles de RNE en 1937.

6 La télévision privée en format hertzien a commencé à émettre en Espagne à partir de 1989 après la promulgation de la Loi de Télévision Privée de cette même année. Les premières concessions de télévision privée ont été pour les chaînes «Tele 5 », « Antena 3 » et « Canal + ». 
à chaque groupe professionnel. Les compléments salariaux seront touchés par le travailleur à condition d'acquérir des connaissances et des compétences grâce aux cours de formation envisagés dans le Plan. Ce nouveau plan de rétribution octroie une plus grande flexibilité et concurrence à l'Organisation.

Dans le cas des candidats destinés à faire partie du «staff » de la direction, la formation et la gestion des talents sont là aussi la clef de leur avancement professionnel. Les travailleurs, à un certain moment, pourraient atteindre d'importants niveaux de responsabilité dans l'organisation et la gestion de l'entreprise grâce à cette proposition fondée sur un système basé sur un Plan de Carrière Professionnelle. Afin d'évaluer les capacités de gestion et d'organisation de ces employés à fort potentiel de direction, on leur proposait l'utilisation de différentes méthodologies basées, surtout, sur l'évaluation $360^{\circ}$, sur l'entrevue par compétence et sur la dénommée « technique d'évaluation du remplissage de fonctions ». De cette façon, la préparation des futurs dirigeants de la Compagnie faisait partie de la stratégie commerciale de la radio-télévision publique.

Suite à la mise en place de ce nouveau système de classification, RTVE a transformé ses anciens profils professionnels et a pu y incorporer d'autres, nouveaux, relatifs aux TIC et aux environnements multimédias ${ }^{7}$. Ainsi, la radio-télévision publique a pu s'adapter à une scène technologique et productive. Les différentes associations professionnelles recommandent l'incorporation de professionnels polyvalents familiarisés avec l'utilisation des nouvelles technologies (Lopez y Ortiz, 2011 et APAR, 2010). En échange, les employés de la radio-télévision publique ont obtenu une réglementation pour leur avancement professionnel, où les règles du jeu sont claires, et où l'effort et l'excellence sont récompensés.

Six ans plus tard, depuis le début de ce nouveau système de classification et d'organisation des Ressources Humaines présenté par la radio-télévision publique, conformément à la planification initialement prévue, ce nouveau modèle devrait déjà être totalement implanté. Mais les changements législatifs postérieurs qui ont réglementé le secteur de la télévision en Espagne tout, comme un certain manque de stabilité des équipes de Direction et de la Présidence de RTVE pendant les dernières six années, ont empêché la complète implantation du projet. Il reste, cependant, des problèmes en suspens : 1'actualisation des Plans de Formation et la reformulation des profils et catégories professionnels pour mieux les adapter au nouveau modèle d'organisation. La solution est proche, dès maintenant, puisque les travailleurs de la RTVE, en septembre 2011, ont ratifié par référendum la totalité du nouveau modèle souscrit par la Direction de 1'entreprise et les représentants syndicaux. Dans ce sens, on peut considérer que cette initiative de la radio-télévision publique reste inachevée.

7 La prestigieuse revue TELOS ( $\left.{ }^{\circ} 8,2011\right)$ publie un article monographique des profils modernes professionnels dans les entreprises de communication. 


\section{La communication interne, outil stratégique pour la mise en œuvre du nouveau modèle d'organisation de RTVE}

Bien que ce nouveau système de classification et d'organisation des Ressources Humaines de RTVE ait été accordé avec les représentants syndicaux, 1'implantation d'une stratégie de communication et sensibilisation afin de transmettre une information positive du contenu aux travailleurs a été nécessaire. L'explication est évidente : on avait besoin de créer une bonne ambiance de travail entre les employés autour du projet de l'entreprise. Contrairement à ce qui se passe dans d'autres secteurs productifs, dans le cas de la stratégie de communication avec les travailleurs, le rôle des syndicats est très important car les deux principales organisations syndicales du pays, CCOO (Comisiones Obreras) et UGT (Unión General de Trabajadores), étaient représentées au Conseil d'Administration de la radio-télévision publique espagnole. Pour ce motif, la stratégie informative s'est effectuée à partir de deux milieux différents : le Département de Communication Interne Corporative et les délégations des Organisations Syndicales des différents centres de travail de la radio-télévision publique (Ortiz, 2012).

$\grave{A}$ cet effet, on a mis en ouvre un système d'information et de communication avec les travailleurs via l'intranet de RTVE, et on a établi des Bureaux d'Information dans chacun des soixante centres de production répartis dans tout le pays. Cette stratégie a été complétée par un plan de sensibilisation destiné au "staff » de la direction. Parallèlement, les syndicats de RTVE ont organisé des journées de sensibilisation et ont créé des Bureaux $\mathrm{d}$ 'Information dans chaque siège syndical. Six ans après sa mise en place, la ratification en référendum du projet patronal par les travailleurs prouve que la stratégie de communication était correcte.

\section{Conclusion}

Les transformations rapides du secteur audiovisuel espagnol ne pouvaient être sans répercussion sur le secteur public. L'environnement médiatique et technologique changeant a obligé les responsables de la radio-télévision publique espagnole à créer une nouvelle gestion des ressources humaines afin d'adapter l'organisation à cette situation. Ce projet avait pour but la recherche d'éléments de promotion et de motivation des travailleurs de la radio-télévision publique, grâce aux Plans de Promotion Professionnelle et de Carrières. C'était le premier modèle d'organisation du secteur de la radio-télévision en Espagne qui mettait en pratique une administration des Ressources Humaines, fondée sur la gestion du talent et la formation permanente des travailleurs. Bien que dans le secteur audiovisuel espagnol, l'initiative soit innovatrice, d'autres fractions du tissu industriel avaient déjà entrepris des initiatives similaires. Concrètement, de grandes corporations appartenant à des secteurs productifs différents avaient déjà mis en fonctionnement, plusieurs années auparavant, les dénommées " universités 
virtuelles corporatives ", avec une orientation semblable au système implanté en RTVE.

Ce nouveau modèle d'organisation et de gestion des ressources humaines de RTVE a compté sur un plan de communication signé entre la direction de l'entreprise et les représentants des travailleurs qui a utilisé des instruments de communication corporative, ainsi que des Bureaux d'Information des organisations syndicales ayant représentation dans la RTVE. Cependant, la proposition, exposée en 2006, ne s'est toujours pas appliquée dans sa totalité. L'actualisation du projet des Plans de Formation et la nouvelle adaptation des profils professionnels aux actuelles nécessités de la radio-télévision publique nationale sont à l'ordre du jour. Un but qui pourrait finalement être atteint à compter de l'approbation par référendum par les travailleurs, l'été 2011, de ce nouveau système de classification et d'organisation. Le développement des questions à traiter devra être accordé au sein d'une commission mixte de suivi : un organe de contrôle bipartite où seront représentées les organisations syndicales et patronales. L'implantation de ce nouveau système de classification et d'organisation des Ressources Humaines dans RTVE a débouché sur un accroissement de sa compétitivité et une adaptation de ses processus productifs au nouveau cadre technologique et médiatique.

\section{BIBLIOGRAPHIE}

APARM, «El audiovisual industria cultural », dans www.aparm.org (15-03-2012). Edt. Asociación de Productores Audiovisuales, España.

IMPULSA TDT., Informe final 2010, 2011, Madrid, Edt. Impulsa TDT. España.

LÓPEZ VIDALES N.Y., ORTIZ SOBRINO M.A. « Perfiles profesionales en Radio », TELOS, n 86, mars 2011, p. 10.

MORILLON L., BOUZON A., COOREN F., " Pour une approche communicationnelle de l'individu au travail ", Communication \& Organisation, $\mathrm{n}^{\circ} 36$, décembre 2009, p. 9.

MURO BENAYAS I., « Crisis de medios y revolución en la cadena de valor noticioso », TELOS, n84, juillet-sep. 2010.

ORTIZ SOBRINO M.A., " Los planes de Carreras y los sistemas de Promoción Profesional como referencia para el nuevo EEES : El caso de la Corporación RTVE », dans El reto en la innovación docente: El EEES como punto de encuentro, D. CALDEVILLA Ed., 2012, Madrid, Visión Libros.

ORTIZ SOBRINO M.A., «Impacto de la creación de la Corporación RTVE en su política de recursos humanos ", Vivat Academia, no 117, février 2012.

RODRIGUEZ BARBA D., PEINADO F., « Nuevos modelos de negocio para la radio ", dans Radio 3.0: Una nueva radio para una nueva era. ORTIZ y LÓPEZ Ed., 2012, Madrid, Ed. Fragua.

SALAVERRÍA R., "Convergencia de Medios ", dans www.Chasqui.comunica.org. (18/03/ 2011). 
SCOLARI C.A., «Alrededor de la convergencia: conversaciones teóricas, divergencias conceptuales y transformaciones en el ecosistema de medios ", Signo y Pensamiento, no 54, volume XXVIII, janvier-juin 2009.

VIVAR, H. Edt., « Nuevos perfiles profesionales para la comunicación digital », TELOS, n ${ }^{\circ} 87,2011$.

Legislación

BOE. Estatuto de la Radio y la Televisión. Madrid. BOE 10 janvier 1980.

BOE. Ley 46/1983 del Tercer Canal de Televisión. Madrid. BOE 26 décembre 1983.

BOE. Ley 17/2006 de la radio y la televisión de titularidad estatal, Madrid. BOE 5 juin 2006.

BOE. Ley 46/1983, del Tercer Canal de Televisión. Madrid. BOE de 26 diciembre 1983 CONVENIO COLECTIVO RTVE (XVII) 2006.

Résumé : Le XXI ${ }^{\text {e }}$ siècle a vu naître les plus grandes transformations dans le secteur audiovisuel espagnol. L'impact technologique des TIC dans les systèmes productifs et l'apparition de nouveaux opérateurs dans la structure médiatique ont impulsé la reformulation des entreprises de radio-télévision, particulièrement dans le domaine de la gestion des Ressources Humaines. En Espagne, l'insoutenable système de financement des mass media de propriété publique et la migration de la télévision analogique vers la numérique TDT «Televisión Digital Terrestre », ont contribué et profondément affecté l'évolution de ce paradigme. Pour affronter l'implantation de redéfinition des processus de production et d'organisation, la radio-télévision publique espagnole a mis en œuvre, en 2006, un modèle d'organisation et classification de ses Ressources Humaines basé sur la gestion du talent et la formation permanente dont quelques aspects restent encore à préciser. Pour la première fois, une telle initiative était mise en œuvre par le secteur public de la radio-télévision en Espagne.

Mots-clés : Organisation, Ressources Humaines, Radio-télévision, Talent, Formation Permanente.

Abstract : The century XXI has witnessed major changes in the Spanish audiovisual sector. The technological impact of ICT in production systems and the emergence of new operators in the media structure have led to the reformulation of radio and television companies, especially in regard to their human resources policies. In the Spanish case, the unsustainable financing system of media in public ownership and the migration of analog television to digital terrestrial television have been added other circumstances that have also contributed to the paradigm shift. To address this process of redefinition of production and organizational processes of the new century, the Spanish public broadcaster implemented - in 2006 - a model of organization and classification of Human Resources based on talent management and continuous training of the that are still some aspects to be specified. It was the first time the public radio and television in Spain put in place an initiative of this nature.

Keywords : Organization, Human Resources, Broadcasting, Talent, Continuous training. 
